\title{
MAJORITY DYNAMICS AND THE RETENTION OF INFORMATION
}

\author{
OMER TAMUZ AND RAN J. TESSLER
}

\begin{abstract}
We consider a group of agents connected by a social network who participate in majority dynamics: each agent starts with an opinion in $\{-1,+1\}$ and repeatedly updates it to match the opinion of the majority of its neighbors.

We assume that one of $\{-1,+1\}$ is the "correct" opinion $S$, and consider a setting in which the initial opinions are independent conditioned on $S$, and biased towards it. They hence contain enough information to reconstruct $S$ with high probability. We ask whether it is still possible to reconstruct $S$ from the agents' opinions after many rounds of updates.

While this is not the case in general, we show that indeed, for a large family of bounded degree graphs, information on $S$ is retained by the process of majority dynamics.

Our proof technique yields novel combinatorial results on majority dynamics on both finite and infinite graphs, with applications to zero temperature Ising models.
\end{abstract}

\section{Contents}

1. Introduction

1.1. Definitions and a statement of the problem

1.2. Results

2. Acknowledgments

3. Proofs

3.1. The Lyapunov functional

3.2. No dynamic monopolies on infinite graphs

3.3. Summable edge weightings and slow growth 10

3.4. Combinatorial majority dynamics results 12

3.5. Invariant random subgraphs 13

3.6. Families of uniformly bounded growth graphs 17

3.7. Convergence on a non-slow growth graph 18

References 18

Date: April 30, 2014.

2010 Mathematics Subject Classification. Primary:. Secondary:

Key words and phrases. Social networks, repeated majority.

Omer Tamuz is supported by ISF grant 1300/08, and is a recipient of the Google Europe Fellowship in Social Computing, and this research is supported in part by this Google Fellowship. 


\section{INTRODUCTION}

Consider a group of people (agents) who each carry one of two possible opinions regarding some issue. Each agent forms an initial opinion, and then repeatedly updates it by observing the opinions of its neighbors in a social network.

Many variants of this model have been studied in diverse settings and disciplines such as Economics (e.g. [1, 5]), Statistical Mechanics (e.g. 4, 6, 10]), Computer Science (see a survey by Shah [18), and Mathematics (e.g. 3, 11]). These include variations on how the agents acquire their initial opinions (e.g., deterministically, at random, arbitrarily or through some other process), what they aim to achieve in this process (e.g., rational agents in Economics [1,7, 16, message passing agents in Computer Science [12]), and how they go about updating their opinions in order to achieve this.

A particularly well studied model is that of majority dynamics, in which agents update their opinions to match that of the majority of their neighbors. We choose a setting in which each agent's initial opinion carries some independent information regarding the "true" opinion, and where it is possible to discover this truth with high probability by aggregating the initial opinions. The question we tackle is the following: is information lost through the process of majority dynamics? When can the "truth" be well estimated even after people have exchanged opinions?

These questions were, to our knowledge, first considered in the context of majority dynamics in Mossel, Neeman and Tamuz [15, who gave both positive and negative examples. We extend their work in several directions, as described below.

As part of our analysis we develop a combinatorial tool that is instrumental in studying majority dynamics. Using it, we present a number of purely combinatorial results on this process. These results can be applied directly to what is known as the dynamics of the zero temperature translation-invariant ferromagnetic Ising model, which, on odd degree graphs, is the same as what we below call asynchronous majority dynamics. In particular, Theorem 1.5 gives, for every odd degree lattice of (say) polynomial growth, a constant $C$ such that regardless of the starting configuration, with probability one no site changes its state more than $C$ times.

1.1. Definitions and a statement of the problem. Let $V$ be a finite or countably infinite set of agents. Let $G=(V, E)$, the social network, be an undirected, connected, locally finite graph. We denote the neighbors of $i \in V$ by $N(i)=\{j:(i, j) \in E\}$, and say that $G$ is $d$-bounded degree when $|N(i)| \leq d$ for all $i \in V$.

We denote by $A_{t}^{i} \in\{-1,+1\}$ agent $i$ 's opinion at time $t$. After drawing some initial opinions $\left\{A_{0}^{i}\right\}_{i \in V}$ from a distribution we describe below, the agents proceed to update their opinions using majority dynamics.

We consider two version of majority dynamics, namely a synchronous and an asynchronous one, and prove all our results for both. In the synchronous model, $t$ takes values in the non-negative integers only, and we set

$$
A_{t+1}^{i}=\operatorname{sgn} \sum_{j \in N(i)} A_{t}^{j} .
$$

In the asynchronous model, we equip each agent with an independent, unit rate Poisson clock, and let each agent update its opinions at the times of its clock rings, 
using

$$
A_{t}^{i}=\operatorname{sgn} \sum_{j \in N(i)} A_{t}^{j}
$$

We assume throughout that the degree of every vertex is odd, so that there are no "ties" and $A_{t}^{i} \in\{-1,+1\}$ for all $t$. Alternatively, given a graph with even degrees, one could add $i$ or remove $i$ from $N(i)$ to make $|N(i)|$ odd.

In the asynchronous model, in order to ensure that the model is well defined, we must rule out the possibility that there will occur an infinite sequence of clock ticks $t_{1}>t_{2}>\cdots>0$ such that agent $i$ updates at time $t_{i}$ and $i \in N(i+1)$. To ensure that this occurs with probability zero, it is sufficient to assume that degrees are bounded. We indeed make this assumption throughout, and prove that it is sufficient in Claim 3.5 below.

A classical result 13 is that for finite graphs, in the synchronous model, each agent's opinion either converges, or, from some time on, oscillates between -1 and +1 with period two; that is, each agent's opinion eventually has period at most two. In the asynchronous model all opinions converge for finite graphs.

The same can be shown to hold for bounded degree infinite graphs with sufficiently slow growth. To state this result we shall need some definitions. Denote graph distances by $\rho(\cdot, \cdot)$, let $n_{r}(G, i)=|\{j: \rho(i, j)=r\}|$ be number of vertices at graph distance $r$ from $i$ in $G$. Finally, denote

$$
\mathrm{M}_{d}[G, i]=\sum_{r=0}^{\infty}\left(\frac{d+1}{d-1}\right)^{-r} n_{r}(G, i) ;
$$

the usefulness of this definition will be demonstrated below.

Ginosar and Holzman 8 prove the following result, which is a strengthening of a somewhat weaker claim by Moran [14].

Theorem 1.1 (Moran, Ginosar and Holzman]). Let $G$ be a d-bounded degree graph such that $M_{d}[G, i]<\infty$ for some ( $\leftrightarrow$ all) $i \in V$. Then each agent's opinion eventually has period at most two in the synchronous model, and converges in the asynchronous model.

Note that this is a combinatorial (rather than a probabilistic) result, in the sense that it holds for every set of initial opinions $\left\{A_{0}^{i}\right\}_{i \in V}$, and, in the asynchronous model, for every choice of clock ring times (in which there are no rings at the same time). We prove this result using a novel combinatorial tool, which yields additional insights into this process (see Section 1.2.1). In particular, we bound the number of times that an agent may change its opinion, and show that an agent will never change its opinion if a large enough neighborhood around it agrees with it.

In Section 3.7 we provide an example of a $d$-regular graph for which $\mathrm{M}_{d}[G, i]=$ $\infty$ for all $i \in V$, and for which, in the synchronous model, each agent's opinion eventually has period at most two for any initial set of opinions. This shows that the sufficient condition of Theorem 1.1 is not necessary. Indeed, the existence of a simple, geometrical, necessary and sufficient condition for convergence to period at most two is an interesting open question.

It should be noted that had we allowed even degrees with random tie breaking, the dynamics would have changed dramatically. Tessler and Louidor [19] discuss

\footnotetext{
${ }^{1}$ Note that Moran, as well as Ginosar and Holzman, prove their theorems for the synchronous model; we extend it to the asynchronous model.
} 
the asynchronous model for trees with even degrees larger than two (for which it may be that $\left.\mathrm{M}_{d}[G, i]<\infty\right)$. It is shown there that if the initial opinions are chosen with i.i.d symmetric Bernoulli distribution, then almost surely there exist some agents that change their opinions infinitely many times.

We henceforth consider only slow growth graphs, i.e. graph for which

$$
\mathrm{M}_{d}[G, i]<\infty,
$$

and for which, by Theorem 1.1 we can define random variables $Z^{i}$ taking values in $\{-1,+1\}$ and given by

$$
Z^{i}=\lim _{t \rightarrow \infty} A_{2 t}^{i},
$$

where the limit is taken over $t \in \mathbb{N}$.

We next describe how the agents acquire their initial opinions. Let $S \in\{-1,+1\}$ be the state of the world with $\mathbb{P}[S=-1]=\mathbb{P}[S=1]=\frac{1}{2}$. We think of $A_{t}^{i} \in$ $\{-1,+1\}$ as agent $i$ 's opinion regarding $S$ at time $t$, and draw $\left\{A_{0}^{i}\right\}_{i \in V}$ as follows: We fix some $\frac{1}{2}<p<1$, and let $A_{0}^{i}=S$ with probability $p$ and $A_{0}^{i}=-S$ with probability $1-p$, with the events ' $A_{0}^{i}=S$ ' being independent of each other and of $S$. Note that it follows that the random variables $A_{0}^{i}$ are not independent, but are independent (in fact, i.i.d) conditioned on $S$.

When $|V|$ is finite but large, then $S$ can be estimated with high probability given $\left\{A_{0}^{i}\right\}_{i \in V}$. When $|V|$ is infinite, $S$ can be estimated exactly given $\left\{A_{0}^{i}\right\}_{i \in V}$. Formally, let $|V|=n$, and let

$$
\hat{S}_{0}=\operatorname{sgn} \sum_{i \in V} A_{0}^{i}
$$

Then by the Chernoff bound

$$
\mathbb{P}\left[\hat{S}_{0} \neq S\right] \leq e^{-O(n)} .
$$

For the case that $|V|=\infty$, let

$$
\mathcal{A}_{0}=\sigma\left(\left\{A_{0}^{i}\right\}_{i \in V}\right) .
$$

Here we denote by $\sigma(X)$ the sigma-algebra generated by $X$, a random variable 2 . Then it holds that

$$
\inf _{\hat{S}_{0} \in \mathcal{A}_{0}} \mathbb{P}\left[\hat{S}_{0} \neq S\right]=0
$$

where, by a slight abuse of notation, we say that $\hat{S}_{0} \in \mathcal{A}_{0}$ when $\hat{S}_{0}$ is $\mathcal{A}_{0}$-measurable.

The question that we tackle is the following: when is it the case that $S$ can still be reconstructed from the limiting opinions $\left\{Z^{i}\right\}_{i \in V}$ ? Formally, define $\mathcal{A}_{\infty}=$ $\sigma\left(\left\{Z^{i}\right\}_{i \in V}\right)$, and let the probability of error (in the reconstruction of $S$ using $\left.\left\{Z^{i}\right\}_{i \in V}\right)$ be given by

$$
\delta(G, p)=\inf _{\hat{S} \in \mathcal{A}_{\infty}} \mathbb{P}[\hat{S} \neq S],
$$

where, to remind the reader, $p=\mathbb{P}\left[A_{0}^{i}=S\right]$ is a parameter of our measure $\mathbb{P}$.

We ask the question of whether $\delta(G, p)$ is equal to zero in the case of an infinite graph, or is close to zero in the case of a large finite graph. Formally, let $\left\{G_{n}\right\}_{n \in \mathbb{N}}$

\footnotetext{
${ }^{2}$ This is the smallest sigma-algebra for which $X$ is measurable.
} 
be a sequence of finite graphs such that $\lim _{n}\left|V_{n}\right|=\infty$. For which sequences and values of $p$ is it the case that $\lim _{n} \delta\left(G_{n}, p\right)=0$ ? And for which infinite $G$ does it hold that $\delta(G, p)=0$ ?

Berger [2] gives an example of a sequence of finite graphs with $\lim _{n}\left|V_{n}\right|=\infty$ and such that in each graph there exists a dynamic monopoly of size eighteen: a set $W \subset V$ of eighteen vertices with the property that if $\left\{A_{0}^{j}\right\}_{j \in W}$ are all equal to some $s \in\{-1,+1\}$ then $Z^{i}=s$ for all $i \in V$ (in the synchronous model) 3 . Since $\mathbb{P}\left[A_{0}^{j}=-S \quad \forall j \in W\right]=(1-p)^{18}$, it follows that $\delta\left(G_{n}, p\right) \geq(1-p)^{18}$.

This example involves graphs with increasingly large degrees. We offer the following conjecture (see also [15]).

Conjecture 1.2. Let $\left\{G_{n}\right\}_{n \in \mathbb{N}}$ be a sequence of d-bounded degree finite graphs such that $\lim _{n}\left|V_{n}\right|=\infty$. Then

$$
\lim _{n \rightarrow \infty} \delta\left(G_{n}, p\right)=0
$$

for all $\frac{1}{2}<p<1$.

Mossel, Neeman and Tamuz [15] show that if $\left\{G_{n}\right\}$ is sequence of transitive graphs (that is, graphs in which all nodes play the same role, see Section 1.2.2 below) then $\lim _{n} \delta\left(G_{n}, p\right)=0$. In fact, they show that the same holds under a weaker assumption, namely that each geometric equivalence class is large (again, see Section 1.2.2 for a precise definition). They also show the same for good enough expander graphs.

For infinite graphs $G$, our question is whether $\delta(G, p)=0$. While we provide some positive results below, we do not know the answer to the following seemingly basic question:

Question 1.3. Does there exist an infinite graph $G$ and $\frac{1}{2}<p<1$ such that $\delta(G, p)>0$ ?

We prove the following claim (see [17] for related work).

Theorem 1.4. In the synchronous model, no infinite, locally finite graph has a dynamic monopoly of finite size.

This result suggests that perhaps $\delta(G, p)=0$ for every finite degree, slow growth graph $G$.

\subsection{Results.}

1.2.1. Majority Dynamics. We begin by proving two combinatorial claims regarding majority dynamics. These claims may be of independent interest, but are also useful in proving our main results.

The first result is a quantitative version of Theorem 1.1

Theorem 1.5. Let $G$ be a d-bounded degree graph such that $M_{d}[G, i]<\infty$ for some ( $\leftrightarrow$ all) $i \in V$. Then

(1) In the synchronous model, the number of times $t$ for which $A_{t+1}^{i} \neq A_{t-1}^{i}$ is at most $\frac{d+1}{d-1} \cdot d \cdot M_{d}[G, i]$.

\footnotetext{
${ }^{3}$ We slightly weaken his definition; he requires that $A_{t}^{i}=s$ for all $i$ in some finite time $t$.

${ }^{4}$ Although the proof of this claim is rather straightforward, we have not found it in the literature.
} 
(2) In the asynchronous model, the number of times in which $i$ changes its opinion is at most $\frac{d+1}{d-1} \cdot 2 d \cdot M_{d}[G, i]$.

We would like to thank the anonymous referee for pointing out to us that this theorem can, for the synchronous model, be easily derived from the work of Ginosar and Holzman.

The next result shows that if a sufficiently large neighborhood of $i$ starts with a certain opinion then $i$ will always have this opinion.

Theorem 1.6. Let $G$ be a d-bounded degree graph such that $M_{d}[G, i]<\infty$ for some ( $\leftrightarrow$ all) $i \in V$. Let $r_{0}$ be such that

$$
\frac{d+1}{d-1} \cdot 2 d \cdot \sum_{r>r_{0}}\left(\frac{d+1}{d-1}\right)^{-r} n_{r}(G, i)<1 .
$$

If $A_{T}^{j}=A_{T}^{i}$ for some $T$ and for all $j$ such that $\rho(i, j) \leq r_{0}+2$, then $A_{t}^{i}=A_{T}^{i}$ for all $t>T$.

1.2.2. Invariant random subgraphs. A graph automorphism of $G=(V, E)$ is a bijection $h: V \rightarrow V$ such that $(i, j) \in E \leftrightarrow(h(i), h(j)) \in E$. We denote by $\operatorname{Aut}(G)$ the automorphism group of $G$. Indeed, it is easy to verify that the graph automorphisms of a given graph form a group under composition.

Let $H$ be a subgroup of $\operatorname{Aut}(G)$. We say that $H$ acts transitively on (the vertices of) $G$ when, for every $i, j \in V$ there exists an $h \in H$ such that $h(i)=j$. Equivalently, $H$ acts transitively on $G$ when $V / H$, the set of $H$ orbits of $V$, is a singleton. We say that $H$ acts quasi-transitively on $G$ when $V / H$ is finite. Finally, we say that $G$ is (quasi-) transitive when $\operatorname{Aut}(G)$ acts on it (quasi-) transitively.

A subgraph of $G_{0}=\left(V_{0}, E_{0}\right)$ is a graph $G=(V, E)$ such that $V \subseteq V_{0}$ and $E \subseteq E_{0}$ is a set of edges on $V$. Let $H$ be a subgroup of $\operatorname{Aut}\left(G_{0}\right)$. A random $G_{0}$-subgraph $G$ (that is, a random variable $G$ that takes values in the space of subgraphs of a graph $\left.G_{0}\right)$ is said to have an $H$-invariant distribution if for all $h \in H$ the law of $h(G)$ equals the law of $G$.

Note that $G$ could have nodes with even degrees even when $G_{0}$ has odd degrees. Since we will want to apply majority dynamics to $G$, we add or remove self-loops to $G$ in order to make all degrees odd. This does not affect the fact that $G$ is $H$-invariant; the modified $G$ is $H$-invariant iff the unmodified $G$ was $H$-invariant. This also does not increase any degree beyond what it was on $G_{0}$, since we do not add a self-loop unless we remove another edge.

The following is our main result for this section.

Theorem 1.7. Let $G_{0}$ be a quasi-transitive infinite graph with maximal degree d such that $M_{d}\left[G_{0}, i\right]<\infty$ for some ( $\leftrightarrow$ all) $i \in V_{0}$.

Let $H \leq \operatorname{Aut}\left(G_{0}\right)$ act quasi-transitively on $G_{0}$, and let $G$ be an infinite connected random subgraph of $G_{0}$ with an $H$-invariant distribution. Then

$$
\delta(G, p)=0
$$

almost surely for any $\frac{1}{2}<p<1$.

It follows from Theorem 1.7 that $\delta(G, p)=0$ for any quasi-transitive, slow growth infinite graph $G$ and any $\frac{1}{2}<p<1$. This is already a non-trivial result, which is generalized in Theorem 1.7 . 
1.2.3. Families of uniformly bounded growth graphs. Given $d \geq 3$ and a function $f: \mathbb{N} \rightarrow \mathbb{N}$, let $\mathcal{B}(f, d)$ be the family of $d$-bounded degree graphs $G$ such that $n_{r}(G, i) \leq f(r)$ for all vertices $i$ in $G$ and $r \in \mathbb{N}$.

Reusing the notation $\mathrm{M}_{d}[\cdot]$, let

$$
\mathrm{M}_{d}[f]=\sum_{r=0}^{\infty}\left(\frac{d+1}{d-1}\right)^{-r} \cdot f(r) .
$$

We say that $f: \mathbb{N} \rightarrow \mathbb{N}$ has slow growth if $\mathrm{M}_{d}[f]<\infty$.

Theorem 1.8. Fix an odd $d \geq 3$, and let $f: \mathbb{N} \rightarrow \mathbb{N}$ have slow growth. There exists $a \frac{1}{2}<p_{0}<1$ such that for all $p_{0}<p<1$ and for all sequences of finite graphs $\left\{G_{n}\right\}_{n \in \mathbb{N}}$ in $\mathcal{B}(f, d)$ such that $\lim _{n}\left|V_{n}\right|=\infty$ it holds that

$$
\lim _{n} \delta\left(G_{n}, p\right)=0,
$$

and for all infinite graphs $G \in \mathcal{B}(f, d)$ it holds that

$$
\delta(G, p)=0 .
$$

\section{ACKNowledgments}

We would like to thank Elchanan Mossel for useful discussions and ideas, and the anonymous referee for additional important comments and corrections.

\section{Proofs}

3.1. The Lyapunov functional. Following Goles and Olivos [9], we analyze the process of Majority dynamics using the technique of Lyapunov functionals. In particular, we build and elaborate on the ideas of Ginosar and Holzman [8] to apply these techniques to infinite graphs. We apply them to both the asynchronous and the synchronous model, and use them to prove some additional, general results for majority dynamics on graphs with uniformly bounded growth.

We begin by introducing some non-standard notation which will allow us to simultaneously treat the synchronous and the asynchronous models. We let the symbol $\Delta$ stand for "1" for in synchronous model and for " $d t$ " in the asynchronous model. For the asynchronous model, we denote

$$
A_{t-\Delta}^{i}=A_{t-d t}^{i}=\lim _{t^{\prime} \rightarrow t^{-}} A_{t^{\prime}}^{i}
$$

and

$$
A_{t+\Delta}^{i}=A_{t+d t}^{i}=\lim _{t^{\prime} \rightarrow t^{+}} A_{t^{\prime}}^{i}=A_{t}^{i},
$$

where the last equality follows from Eq. 1.2 The definitions of majority dynamics, Eqs. 1.1 and 1.2, can now be written in one equation:

$$
A_{t+\Delta}^{i}=\operatorname{sgn} \sum_{j \in N(i)} A_{t}^{j} .
$$

Let $G=(V, E)$ be a finite or infinite $d$-bounded degree graph. Let a $d$-legal edge weighting $z: E \rightarrow(0,1]$ be such that for any two adjacent edges $e_{1}=(i, j)$ and $e_{2}=(i, k)$ it holds that

$$
\frac{z\left(e_{1}\right)}{z\left(e_{2}\right)}<1+\frac{2}{d-1}=\frac{d+1}{d-1} .
$$


Note that $z$ is a function on the undirected edge set $E$, and so $z(i, j)=z(j, i)$. It is easy to see that

$$
\operatorname{sgn} \sum_{j \in N(i)} A_{t}^{j}=\operatorname{sgn} \sum_{j \in N(i)} z(i, j) A_{t}^{j} .
$$

Indeed, assume $i$ has $k$ neighbors, and that the majority of their opinions are, without loss of generality, +1 . Then $i$ has at least $\frac{k+1}{2}$ neighbors with opinion +1 . Denote by $N_{+}$this set of neighbors. Similarly, $i$ has at most $\frac{k-1}{2}$ neighbors with opinion -1 , which we denote by $N_{-}$. Let $z_{1}$ be the largest $z(i, j)$ for $j \in N(i)$, and $z_{2}$ be the smallest $z(i, j)$ in the same set. By assumption, and because $k \leq d$,

$$
\frac{z_{1}}{z_{2}}<\frac{d+1}{d-1} \leq \frac{k+1}{k-1}
$$

Hence,

$$
\begin{aligned}
\sum_{j \in N(i)} z(i, j) A_{t}^{j} & \geq z_{2} \sum_{j \in N_{+}} 1-z_{1} \sum_{j \in N_{-}} 1 \\
& =z_{2}\left(\left|N_{+}\right|-\frac{z_{1}}{z_{2}}\left|N_{-}\right|\right) \\
& \geq z_{2}\left(\frac{k+1}{2}-\frac{z_{1}}{z_{2}} \frac{k-1}{2}\right),
\end{aligned}
$$

the last expression is positive due to the second inequality in the previous display, and Eq. 3.2 follows. Thus, the definitions of majority dynamics (Eqs. 1.1 and 1.2) can equivalently be written as

$$
A_{t+\Delta}^{i}=\operatorname{sgn} \sum_{j \in N(i)} z(i, j) A_{t}^{j} .
$$

Yet another equivalent definition is

$$
A_{t+\Delta}^{i}=\underset{a \in\{-1,+1\}}{\operatorname{argmin}} \sum_{j \in N(i)} z(i, j)\left(A_{t}^{j}-a\right)^{2} ;
$$

intuitively, each agent in each turn can be seen as trying to minimize the "energy" $\sum_{j \in N(i)} z(i, j)\left(A_{t}^{j}-a\right)^{2}$. This motivates the following definition:

$$
L_{t}=\frac{1}{4} \sum_{(i, j) \in E} z(i, j)\left(A_{t+\Delta}^{i}-A_{t}^{j}\right)^{2} .
$$

Of course, $L_{t}$ has to be finite for this definition to be useful, and we indeed give in Proposition 3.4 a necessary and sufficient geometrical condition under which a $d$-legal summable $z$ exists. Note also that $L_{t}$ depends (implicitly) on the choice of $z$.

It turns out that this definition of $L_{t}$ is a correct choice for a "Lyapunov functional", in the sense that $L_{t}$ is monotone non-increasing.

Proposition 3.1. $L_{T+t} \leq L_{T}$ for all $T, t \geq 0$.

Before proving this proposition, we will need the following definitions and lemma. Define $J_{t}^{i}$ by

$$
J_{t}^{i}=\frac{1}{2}\left(A_{t+\Delta}^{i}-A_{t-\Delta}^{i}\right) \sum_{j \in N(i)} z(i, j) A_{t}^{j},
$$


and let

$$
J_{t}=\sum_{i \in V} J_{t}^{i}
$$

Claim 3.2. $J_{t}^{i} \geq 0$, and $J_{t}^{i}=0$ iff $A_{t+\Delta}^{i}=A_{t-\Delta}^{i}$.

Proof. Since we assume that $|N(i)|$ is odd, $\sum_{j \in N(i)} z(i, j) A_{t}^{j}$ is never zero. It follows that $J_{t}^{i}=0$ iff $A_{t+\Delta}^{i}=A_{t-\Delta}^{i}$.

To see that $J_{t}^{i} \geq 0$, note that when $A_{t+\Delta}^{i}=A_{t-\Delta}^{i}$ then $J_{t}^{i}=0$. Otherwise we have that $J_{t}^{i}=A_{t+\Delta}^{i} \sum_{j \in N(i)} z(i, j) A_{t}^{j}$. But $A_{t+\Delta}^{i}=\operatorname{sgn} \sum_{j \in N(i)} z(i, j) A_{t}^{j}$ and so $J_{t}^{i}$ is equal to the product of two equal sign multiplicands and is therefore positive.

\section{Claim 3.3.}

$$
L_{t}-L_{t-\Delta}=-J_{t}
$$

Proof.

$$
\begin{aligned}
L_{t}-L_{t-\Delta} & =\frac{1}{4} \sum_{(i, j) \in E} z(i, j)\left[\left(A_{t+\Delta}^{i}-A_{t}^{j}\right)^{2}-\left(A_{t}^{i}-A_{t-\Delta}^{j}\right)^{2}\right] \\
& =-\frac{1}{2} \sum_{(i, j) \in E} z(i, j)\left[A_{t+\Delta}^{i} A_{t}^{j}-A_{t}^{i} A_{t-\Delta}^{j}\right]
\end{aligned}
$$

Since the edges are undirected, summing over $(i, j) \in E$ is the same as summing over $(j, i) \in E$. Therefore, and since $z(i, j)=z(j, i)$, we can exchange the roles of $i$ and $j$ in the last summand:

$$
\begin{aligned}
& =-\frac{1}{2} \sum_{(i, j) \in E} z(i, j)\left[A_{t+\Delta}^{i} A_{t}^{j}-A_{t}^{j} A_{t-\Delta}^{i}\right] \\
& =-\sum_{i \in V} \frac{1}{2}\left(A_{t+\Delta}^{i}-A_{t-\Delta}^{i}\right) \sum_{j \in N(i)} z(i, j) A_{t}^{j} \\
& =-\sum_{i \in V} J_{t}^{i} \\
& =-J_{t} .
\end{aligned}
$$

The proof of Proposition 3.1 is now immediate.

Proof of Proposition 3.1. Since $L_{t}-L_{t-\Delta}=-J_{t}$ and since $J_{t} \geq 0$, it follows that $L_{T+t} \leq L_{T}$ for all $T, t \geq 0$.

3.2. No dynamic monopolies on infinite graphs. As a simple application of Claims 3.2 and 3.3 we show that infinite graphs cannot have dynamic monopolies.

Proof of Theorem 1.4. Consider the synchronous model. Let $G$ be an infinite graph, and let $z: E \rightarrow \mathbb{R}^{+}$be the $d$-legal edge weighting given by the constant function $z=1$. Then $z$ is not summable, but Claims 3.2 and 3.3 still hold, given that initial signals are chosen so that $L_{t}$ is finite.

Let $W$ be a finite set of vertices. Let $A_{0}^{i}=+1$ for all $i \in W$ and $A_{0}^{i}=-1$ for all $i \notin W$. Then $L_{0}$ is finite. Note that with $z$ constant, $L_{t}$ and $J_{t}^{i}$ are integer, and therefore $L_{t}$ decreases by at least one whenever $A_{t+1}^{i} \neq A_{t-1}^{i}$. Since $L_{t} \geq 0$, at 
most a finite number of agents change their opinion to +1 , and $W$ is not a dynamic monopoly.

It may be possible to give a stronger, quantitative version of this theorem, by using an edge weighting that increases with the distance from $W$.

3.3. Summable edge weightings and slow growth. We are now almost ready to prove Theorem 1.1. Before that, we will show that graphs with slow growth admit summable, $d$-legal edge weightings.

Proposition 3.4. Let $G=(V, E)$ be a d-bounded degree graph. Then the following are equivalent.

(1) $G$ admits a summable, d-legal edge weighting.

(2) $M_{d}[G, i]<\infty$ for some ( $\leftrightarrow$ all) $i \in V$.

Proof. Let $G$ be a $d$-bounded degree graph, and denote $a=(d+1) /(d-1)$. For a node $i$ and an edge $e=(j, k)$, denote by $\rho(i, e)=\min \{\rho(i, j), \rho(i, k)\}$ the distance of $e$ from $i$.

We first show that (1) implies (2). Let $G$ admit a summable, $d$-legal edge weighting $z$. Let $e_{0}=(i, j)$ an edge. By the definition of $d$-legal edge weightings (Eq. 3.1), given an edge $e$ such that $\rho(i, e)=r$, we have that

$$
z(e) \geq z\left(e_{0}\right) a^{-r-1} \text {. }
$$

Hence, by the fact that $z$ is summable, it follows that

$$
\infty>\sum_{e \in E} z(e)=\sum_{r=0}^{\infty} \sum_{\{e \in E: \rho(i, e)=r\}} z(e) \geq \frac{z\left(e_{0}\right)}{a} \sum_{r=0}^{\infty} a^{-r}|\{e \in E: \rho(i, e)=r\}| .
$$

Now, the number of edges at distance $r$ is greater than or equal to the number of nodes at distance $r+1, n_{r+1}(G, i)$. Hence

$$
\begin{aligned}
\infty & >\frac{z\left(e_{0}\right)}{a} \sum_{r=0}^{\infty} n_{r+1}(G, i) a^{-r} \\
& =z\left(e_{0}\right) \sum_{r=1}^{\infty} n_{r}(G, i) a^{-r} \\
& =z\left(e_{0}\right)\left(\mathrm{M}_{d}[G, i]-1\right),
\end{aligned}
$$

where the last equality follows from the definition of $\mathrm{M}_{d}[G, i]$ and the fact that $n_{0}(G, i)=1$. Thus $\mathrm{M}_{d}[G, i]$ is finite.

We now show that (2) implies (1). Let $\mathrm{M}_{d}[G, i]<\infty$ for some $i \in V$. Let $\eta: \mathbb{N} \rightarrow(1 / a, 1)$ be any monotone increasing function, and let

$$
z(e)=a^{-\rho(i, e)} \frac{\eta(\rho(i, e))}{\eta(0)}
$$

be an edge weighting. We will show that it is $d$-legal and summable. Indeed,

$$
\begin{aligned}
\sum_{e \in E} z(e) & =\sum_{r=0}^{\infty} a^{-r} \frac{\eta(r)}{\eta(0)}|\{e \in E: \rho(i, e)=r\}| \\
& \leq \sum_{r=0}^{\infty} a^{-r} \frac{\eta(r)}{\eta(0)} \cdot d \cdot|\{j \in V: \rho(i, j)=r\}|
\end{aligned}
$$


since the number of edges at distance $r$ is at most $d$ times the number of vertices at that distance. Since $n_{r}(G, i)=|\{j \in V: \rho(i, j)=r\}|$ and since $\eta(r)<1$ then

$$
\begin{aligned}
& <\frac{d}{\eta(0)} \sum_{r=0}^{\infty} a^{-r} \cdot n_{r}(G, i) \\
& =\frac{d}{\eta(0)} \cdot \mathrm{M}_{d}[G, i] \\
& <\infty,
\end{aligned}
$$

and so $z$ is summable.

To see that $z$ is $d$-legal, note that if $e_{1}$ and $e_{2}$ are adjacent then either

$$
\rho\left(i, e_{1}\right)=\rho\left(i, e_{2}\right)
$$

in which case $z\left(e_{1}\right)=z\left(e_{2}\right)$, or else, without loss of generality

$$
\rho\left(i, e_{2}\right)=\rho\left(i, e_{1}\right)+1
$$

In this case, denoting $r=\rho\left(i, e_{1}\right)$,

$$
\frac{z\left(e_{2}\right)}{z\left(e_{1}\right)}=\frac{a^{-r-1} \eta(r+1)}{a^{-r} \eta(r)}=a^{-1} \frac{\eta(r+1)}{\eta(r)}<1,
$$

where the last inequality follows from the fact that $\eta(r+1)<1$ and $\eta(r)>1 / a$. Likewise,

$$
\frac{z\left(e_{1}\right)}{z\left(e_{2}\right)}=\frac{a^{-r} \eta(r)}{a^{-r-1} \eta(r+1)}=a \frac{\eta(r)}{\eta(r+1)}<a=\frac{d+1}{d-1}
$$

where the last inequality follows from the fact that $\eta$ is monotone increasing.

Proof of Theorem 1.1. Let $z$ be a $d$-legal summable edge weighting, as defined in the proof of Proposition 3.4. Note that $L_{t}$ is non-negative, and also finite for all $t$ :

$$
\begin{aligned}
L_{t} & =\frac{1}{4} \sum_{(i, j) \in E} z(i, j)\left(A_{t+\Delta}^{i}-A_{t}^{j}\right)^{2} \\
& \leq \sum_{(i, j) \in E} z(i, j) .
\end{aligned}
$$

Since $L_{t}$ is finite, non-negative and non-increasing, it follows that

$$
L=\lim _{t \rightarrow \infty} L_{t}
$$

always exists and is non-negative.

Fix the initial opinions, the times of the clock rings (for the asynchronous model) and a vertex $j$. Note that $J_{t}^{j}$ is either zero or greater than some $\epsilon_{j}>0$. Let $T$ be such that $L_{T}-L<\epsilon_{j}$. It then follows from Claims 3.2 and 3.3 that $J_{t}^{j}=0$ for all $t>T$, since otherwise it would be the case that $J_{t}^{j}>\epsilon_{j}$ and $L_{t+\Delta}<L$. Now, by Claim 3.2 it follows from this that $A_{t+\Delta}^{j}=A_{t-\Delta}^{j}$ for all $t>T$, and so $j$ 's opinion has period at most two in the synchronous case, and converges in the asynchronous case. 
3.4. Combinatorial majority dynamics results. Let $G=(V, E)$ be a $d$-bounded degree graph such that $\mathrm{M}_{d}[G, i]<\infty$ for some $i \in V$. As per the proof of Proposition 3.4. let $z$ be a summable, $d$-legal edge weighting given by

$$
z(e)=a^{-\rho(i, e)} \frac{\eta(\rho(i, e))}{\eta(0)},
$$

where $a=(d+1) /(d-1)$, and $\eta: \mathbb{N} \rightarrow(1 / a, 1)$ is some monotone increasing function. In fact, by the proof of Proposition 3.4, we have that

$$
\sum_{e \in E} z(e) \leq \frac{d}{\eta(0)} \cdot \mathrm{M}_{d}[G, i] \leq \frac{d+1}{d-1} \cdot d \cdot \mathrm{M}_{d}[G, i] .
$$

Proof of Theorem 1.5. By Claim 3.2, the number of times that $A_{t+\Delta}^{i} \neq A_{t-\Delta}^{i}$ is equal to the number of times that $J_{t}^{i} \neq 0$. Hence we will prove the claim by bounding the number of times that $J_{t}^{i} \neq 0$.

Since $z(i, j)=1$ for all $j \in N(i), J_{t}^{i} \geq 1$ whenever $J_{t}^{i} \neq 0$. Since $L=\lim _{t \rightarrow \infty} L_{t}$ is non-negative, and since $L_{t}-L_{t-\Delta}=-J_{t}$ by Claim 3.3 , it follows that the number of times that $J_{t}^{i} \neq 0$ is at most $L_{0}$.

Now, in the asynchronous case

$$
\begin{aligned}
L_{0} & =\frac{1}{4} \sum_{(k, j) \in E} z(k, j)\left(A_{0}^{k}-A_{0}^{j}\right)^{2} \\
& \leq 2 \sum_{e \in E} z(e) \\
& \leq \frac{d+1}{d-1} \cdot 2 d \cdot \mathrm{M}_{d}[G, i] .
\end{aligned}
$$

In the synchronous case this bound can be improved. By Eq. 3.3, we have that

$$
\frac{1}{4} \sum_{j \in N(k)} z(j, k)\left(A_{1}^{k}-A_{0}^{j}\right)^{2}<\frac{1}{2} \sum_{j \in N(k)} z(j, k) .
$$

Hence

$$
\begin{aligned}
L_{0} & =\frac{1}{4} \sum_{(k, j) \in E} z(k, j)\left(A_{1}^{k}-A_{0}^{j}\right)^{2} \\
& <\sum_{e \in E} z(e) \\
& \leq \frac{d+1}{d-1} \cdot d \cdot \mathrm{M}_{d}[G, i] .
\end{aligned}
$$

Proof of Theorem 1.6. Let $r_{0}$ be such that

$$
\frac{d+1}{d-1} \cdot 2 d \cdot \sum_{r>r_{0}}\left(\frac{d-1}{d+1}\right)^{r} \cdot n_{r}(G, i)<1 .
$$

Then it is easy to verify that

$$
\sum_{e \in E} \mathbb{1}_{\rho(i, e)>r_{0}} z(e)<\frac{1}{2} .
$$


If $A_{T}^{j}=s$ for all $j$ within distance $r_{0}+2$ from $i$, then $A_{T+\Delta}^{j}=s$ for all $j$ within distance $r_{0}+1$ from $i$, and $A_{T+\Delta}^{k}=s$ for all $e=(j, k)$ with $\rho(i, e) \leq r_{0}$. Hence

$$
\begin{aligned}
L_{T} & =\frac{1}{4} \sum_{(k, j) \in E} z(k, j)\left(A_{T+\Delta}^{k}-A_{T}^{j}\right)^{2} \\
& \leq 2 \sum_{e \in E} \mathbb{1}_{\rho(i, e)>r_{0}} z(e) \\
& <1 .
\end{aligned}
$$

Now, as in the proof of Theorem 1.5 above, if $J_{t}^{i} \neq 0$ then $J_{t}^{i} \geq 1$. However, since $L_{T}<1$, since $L_{t}-L_{t-\Delta}=-J_{t}$, and since $L_{t}$ is non-negative, it follows that $J_{t}^{i}=0$ for all $t>T$, and so, in particular, $A_{t+\Delta}^{i}=A_{t-\Delta}^{i}$ for all $t>T$. This completes the proof for the asynchronous model. In the synchronous model, $A_{T}^{i}=A_{T+1}^{i}$, since all of $i$ 's neighbors also have $A_{T}^{j}=s$, and so it follows that $A_{T}^{i}=s$ for all $t>T$.

\subsection{Invariant random subgraphs.}

3.5.1. Light cones. We commence by defining for each vertex $i$ and time $t$ the set of vertices $C_{t}^{i}$ that form $i$ 's past light cone at time $t$. This is the set of vertices whose initial opinions may have influenced $i$ 's opinion at time $t$. Formally, set $C_{0}^{i}=\{i\}$ for all $i \in V$. At every time $t$ in which $i$ updates its opinion, update $C_{t}^{i}$ by

$$
C_{t}^{i}=\bigcup_{j \in N(i)} C_{t-\Delta}^{j}
$$

In the synchronous model, $C_{t}^{i}$ is simply the ball of radius $t$ around $i$. In the asynchronous model every vertex is a member of $C_{t}^{i}$ with positive probability. However $C_{t}^{i}$ is still finite with probability one.

Claim 3.5. Let $G$ be a d-bounded degree graph. Then for all $i \in V$ and $t \geq 0$ it holds that $\mathbb{P}\left[\left|C_{t}^{i}\right|<\infty\right]=1$.

It follows that the asynchronous model is well defined, since if there exists an infinite sequence of times $t_{1}>t_{2}>\cdots>0$ such that $i$ updates at time $t_{i}$ and $i \in N(i+1)$, then $\left|C_{t_{1}}^{1}\right|=\infty$.

Proof. Note that $\left|C_{t}^{i}\right|$ is stochastically dominated from above by the total number of offsprings in a Galton-Watson process, where each vertex has $d$ children with probability equal to the probability of a clock ring in $[0, t]$, and zero children otherwise. Since, for $t$ small enough, the expected number of children in this process is lower than one, it follows that the total number of offsprings is almost surely finite. Hence for $t$ small enough (e.g., $1 /(100 d)$ ) we have that $\mathbb{P}\left[\left|C_{t}^{i}\right|=\infty\right]=0$.

Now, the event that $\left|C_{t}^{1}\right|=\infty$ is equivalent to the existence infinite sequence of times $t_{1}>t_{2}>\cdots>0$ such that (after an appropriate renaming of the vertices) $i$ updates at time $t_{i}$ and $i \in N(i+1)$. By the time-shift-invariance of the clock tick process we can assume that $\lim _{i} t_{t}=0$, and so, if $\mathbb{P}\left[\left|C_{t}^{1}\right|=\infty\right]>0$ for some $t$ then $\mathbb{P}\left[\left|C_{1 /(100 d)}^{j}\right|=\infty\right]>0$ for some $j$. But this is false by the above, and so $\mathbb{P}\left[\left|C_{t}^{i}\right|=\infty\right]=0$ for all $i$ and all $t$.

We say that vertices $i$ and $j$ are causally connected at time $t$ if $C_{t}^{i} \cap C_{t}^{j} \neq \emptyset$. The following is immediate. 
Claim 3.6. Condition on the event that the clock ring times are such that $i$ and $j$ are not causally connected at time $t$. Then the events ' $A_{t}^{i}=S$ ' and ' $A_{t}^{j}=S$ ' are independent.

3.5.2. Choosing independent vertices. We next proceed to construct, for each time $t$, a set of vertices whose opinions at time $t$ are close to being independent, conditioned on $S$.

Let $G=(V, E)$ be an infinite quasi-transitive graph. Then it is standard to show that given $\delta>0$ and a positive integer $t$, one may find a number $r_{t, \delta}$ such that, if $i, j$ are two vertices whose distance is more than $r_{t, \delta}$, then the probability that $i$ and $j$ are causally connected at time $t$ (meaning $C_{t}^{i} \cap C_{t}^{j} \neq \emptyset$ ) is at most $\delta$. Note that in the synchronous case one can take $r_{t, \delta}=r_{t, 0}=2 t$.

For every time $t$ and $\delta>0$, let $W_{t, \delta} \subseteq V$ be a random subset of $V$, drawn as follows. Associate to each vertex in $i \in V$ an i.i.d. exponential random variable $X_{i}$. A vertex $i$ belongs to $W_{t, \delta}$ if and only if $X_{i}<X_{j}$ for all $j$ with $\rho(i, j) \leq r_{t, \delta}$.

We note a number of easily verifiable facts regarding $W_{t, \delta}$.

(1) Any two elements of $W_{t, \delta}$ are at distance more than $r_{t, \delta}$. Hence, by the definition of causal connectedness, any two vertices in $W_{t, \delta}$ are causally connected with probability at most $\delta$.

(2) For all $h \in \operatorname{Aut}(G)$ it holds that $h\left(W_{t, \delta}\right)$ has the same law as $W_{t, \delta}$; that is, the distribution of $W_{t, \delta}$ is $\operatorname{Aut}(G)$-invariant.

(3) $W_{t, \delta}$ is almost surely infinite, since in quasi-transitive graphs the size of radius $r$ balls is uniformly bounded.

If furthermore $H \leq \operatorname{Aut}(G)$ acts quasi-transitively on $G$ then $W_{t, \delta}$ intersects each orbit $V / H$ infinitely often.

3.5.3. Convergence and uniform convergence to $Z$. Let $G=(V, E)$ be a slow-growth graph. Then, as we show above, the limit $Z^{i}=\lim _{t} A_{2 t}^{i}$ exists almost surely. Hence for each vertex $i$ the function $q_{i}$ given by $q_{i}(2 t)=\mathbb{P}\left[Z^{i} \neq A_{2 t}^{i}\right]$ converges to zero.

Clearly, if $i$ and $j$ are both in the same orbit $V / \operatorname{Aut}(G)$, then $q_{i}=q_{j}$. It follows that for every quasi-transitive graph, that is, for every graph with a finite number of orbits, there exists a single function $q$ that converges to zero and such that $q(2 t) \geq \mathbb{P}\left[Z^{i} \neq A_{2 t}^{i}\right]$ for all $i \in V$. In this case we say that we have uniform convergence to $Z^{i}$. The next claim states that the same holds on a shift-invariant random graph chosen from a quasi-transitive graph.

Claim 3.7. Let $G_{0}$ be a quasi-transitive infinite graph with maximal degree d such that $M_{d}\left[G_{0}, i\right]<\infty$ for some ( $\leftrightarrow$ all) $i \in V_{0}$.

Let $H \leq \operatorname{Aut}\left(G_{0}\right)$ act quasi-transitively on $G_{0}$, and let $G$ be an infinite random subgraph of $G_{0}$ with an $H$-invariant distribution. Then there exists a function $q: 2 \mathbb{N} \rightarrow[0,1]$ with $\lim _{n} q(2 n)=0$ such that $q(2 t) \geq \mathbb{P}\left[Z^{i} \neq A_{2 t}^{i} \mid i \in G\right]$.

Proof. Let $i, j \in V_{0}$ belong to the same orbit in $V / H$, so that there exists an $h \in H$ such that $h(i)=j$.

Since $G$ is $h$-invariant, it is possible, using $h$, to couple two copies of our probability space in such a way that $A_{t}^{i}$ in the first copy equals $A_{t}^{j}$ in the second for all $t, Z^{i}$ in the first copy equals $Z^{j}$ in the second, and furthermore $i \in G$ in the first copy iff $j \in G$ in the second. It follows that

$$
\mathbb{P}\left[Z^{i} \neq A_{2 t}^{i} \mid i \in G\right]=\mathbb{P}\left[Z^{j} \neq A_{2 t}^{j} \mid j \in G\right] .
$$


Let $\left(i_{1}, \ldots, i_{k}\right)$ be representatives of the orbits $V / H$. Then

$$
q(2 t)=\max _{k}\left\{\mathbb{P}\left[Z^{i_{k}} \neq A_{2 t}^{i_{k}} \mid i_{k} \in G\right]\right\}
$$

satisfies the conditions of the claim.

3.5.4. Personal estimates of $S$. Before proceeding to show that $S$ can be well estimated given $\left\{Z^{i}\right\}_{i \in V}$, we note that each agent's opinion is always equal to $S$ with probability at least $p$. This is true, by definition, at time $t=0$, and it may not be surprising that this is also the case in later times. However, the proof of this fact is not completely straightforward.

We show in Claim 3.5 above that in the asynchronous case, with probability one there does not exist an infinite sequence of times $t_{1}>t_{2}>\cdots>0$ such that $i$ updates at time $t_{i}$ and $i \in N(i+1)$. Let $\bar{T}$ denote an arbitrary choice of clock ring times for which indeed such a chain does not exist. In the synchronous case let $\bar{T}$ be a trivial (probability one) event.

Claim 3.8. For any $\bar{T}$, agent $i$ and time $t$ it holds that

$$
\mathbb{P}\left[A_{t}^{i}=S \mid \bar{T}\right] \geq p .
$$

Proof. Conditioned on $\bar{T}, C_{t}^{i}$ is fixed and so $A_{t}^{i}$ is a deterministic function of $\left\{A_{0}^{j}\right.$ : $\left.j \in C_{t}^{i}\right\}$. Let $k=\left|C_{t}^{i}\right|$, and denote this function by $f:\{-1,+1\}^{k} \rightarrow\{-1,+1\}$.

Clearly, $f$ is monotone, in the sense that

$$
f\left(x_{1}, \ldots, x_{i-1}, x_{i}, x_{i+1}, \ldots, x_{k}\right) \leq f\left(x_{1}, \ldots, x_{i-1},+1, x_{i+1}, \ldots, x_{k}\right),
$$

and is symmetric in the sense that

$$
f\left(-x_{1},-x_{2}, \ldots,-x_{k}\right)=-f\left(x_{1}, x_{2}, \ldots, x_{k}\right) .
$$

It then follows from Lemma 6.1 in [15] that

$$
\mathbb{P}\left[A_{t}^{i}=+1 \mid \bar{T}, S=+1\right] \geq p,
$$

and so, unconditioned on $S$, it holds that

$$
\mathbb{P}\left[A_{t}^{i}=S \mid \bar{T}\right] \geq p .
$$

3.5.5. Estimating $S$. As in the setting of Theorem 1.7 let $G_{0}$ be a quasi-transitive infinite graph with maximal degree $d$ such that $\mathrm{M}_{d}\left[G_{0}, i\right]<\infty$ for some ( $\leftrightarrow$ all) $i \in V_{0}$. Pick the sets $W_{t, \delta}$ from $V_{0}$, as described above in Section 3.5.2.

Let $H \leq \operatorname{Aut}\left(G_{0}\right)$ act transitively on $G_{0}$, and let $G=(V, E)$ be an infinite connected random subgraph of $G_{0}$ with an $H$-invariant distribution. Let $V_{t, \delta}=$ $W_{t, \delta} \cap V$. Note that $W_{t, \delta}$ and $V$ are independent and are both infinite and $H$ invariant. Furthermore, as we note above, $W_{t, \delta}$ intersects every orbit $V / \operatorname{Aut}\left(G_{0}\right)$ infinitely. It follows that $V_{t, \delta}$ is almost surely infinite.

Claim 3.9. Fix an enumeration of the vertices of $G_{0}$, let $\left\{i_{1}, i_{2}, \ldots\right\}$ be the induced enumeration of the vertices in $V_{t, \delta}$, and let

$$
\hat{S}_{t, \delta}^{n}=\operatorname{sgn} \frac{1}{n} \sum_{k=1}^{n} A_{t}^{i_{k}} .
$$

Then

$$
\mathbb{P}\left[\hat{S}_{t, \delta}^{n} \neq S\right] \leq e^{-\frac{(p-1 / 2)^{2}}{2 p} n}+n^{2} \delta .
$$


Proof. Let $E$ be the event that the clock ring times are such that $\left\{i_{1}, \ldots, i_{n}\right\}$ are not causally connected at time $t$. This happens with probability at least $1-n^{2} \delta$. Then conditioned on $E$, by Claim [3.6] the events ' $A_{t}^{i_{k}}=S$ ' are independent.

Now, by Claim 3.8 , for all $i$ and $t$ it holds that $\mathbb{P}\left[A_{t}^{i}=S \mid E\right] \geq p$. Hence the claim follows by the Chernoff bound.

We are now ready to prove Theorem 1.7

Proof of Theorem 1.7. We will prove the theorem by showing that for every $\epsilon>0$ there exists an $\hat{S}_{\epsilon}$ that is $\mathcal{A}_{\infty}$-measurable and such that

$$
\mathbb{P}\left[\hat{S}_{\epsilon} \neq S\right] \leq \epsilon
$$

Let $N$ and $\delta$ be such that for every $t$ it holds that

$$
\mathbb{P}\left[\hat{S}_{t, \delta}^{N} \neq S\right] \leq \epsilon / 2
$$

where, as in Claim 3.9, $\hat{S}_{t, \delta}^{N}$ is given by

$$
\hat{S}_{t, \delta}^{N}=\operatorname{sgn} \frac{1}{N} \sum_{k=1}^{N} A_{t}^{i_{k}},
$$

with $\left\{i_{1}, i_{2}, \ldots\right\}$ an enumeration of the vertices in $V_{t, \delta}$ which is induced by a fixed enumeration of the vertices of $G_{0}$. The existence of such $N$ and $\delta$ satisfying Eq. 3.4 is guaranteed by Claim 3.9 .

Let $q$ be a function which satisfies

$$
q(2 t) \geq \mathbb{P}\left[Z^{i} \neq A_{2 t}^{i}\right], \lim _{t \rightarrow \infty} q(t)=0,
$$

as given in Claim 3.7. Let $T \in 2 \mathbb{N}$ be such that $q(T) \leq \epsilon /(2 N)$, and define $\hat{S}_{\epsilon}$ by

$$
\hat{S}_{\epsilon}=\operatorname{sgn} \frac{1}{N} \sum_{k=1}^{N} Z^{i_{k}},
$$

where $\left\{i_{1}, i_{2}, \ldots\right\}$ is the same enumeration used to define $\hat{S}_{T, \delta}^{N}$. Then

$$
\mathbb{P}\left[\hat{S}_{\epsilon} \neq \hat{S}_{T, \delta}^{N}\right] \leq \mathbb{P}\left[Z^{i_{k}} \neq A_{T}^{i_{k}} \text { for some } 1 \leq k \leq N\right] .
$$

Now,

$$
\mathbb{P}\left[Z^{i} \neq A_{T}^{i} \mid i \in V_{t, \delta}\right]=\mathbb{P}\left[Z^{i} \neq A_{T}^{i}\right] \leq q(T) .
$$

The equality holds since the choice of $V_{t, \delta}$ is independent of the majority dynamics process, and the inequality is simply a reference to the definition of $q(\cdot)$.

Hence by the union bound we have that

$$
\mathbb{P}\left[Z^{i_{k}} \neq A_{T}^{i_{k}} \text { for some } 1 \leq k \leq N\right] \leq N \cdot q(T) \leq \epsilon / 2,
$$

and so

$$
\mathbb{P}\left[\hat{S}_{\epsilon} \neq \hat{S}_{T, \delta}^{N}\right] \leq \epsilon / 2 .
$$

Combining this with Eq. 3.4, we have that

$$
\mathbb{P}\left[\hat{S}_{\epsilon} \neq S\right] \leq \epsilon
$$


3.6. Families of uniformly bounded growth graphs. Recall that given $d \geq 3$ and a function $f: \mathbb{N} \rightarrow \mathbb{N}, \mathcal{B}(f, d)$ is the family of $d$-bounded degree graphs $G$ such that $n_{r}(G, i) \leq f(r)$ for all vertices $i$ in $G$ and $r \in \mathbb{N}$. Recall also that we say that $f: \mathbb{N} \rightarrow \mathbb{N}$ has slow growth if $\mathrm{M}_{d}[f]<\infty$.

Fix $d \geq 3$, and let $f$ have slow growth. Let $r_{0}$ be the smallest integer for which it holds that

$$
\frac{d+1}{d-1} \cdot 2 d \cdot \sum_{r>r_{0}}\left(\frac{d-1}{d+1}\right)^{r} \cdot f(r)<1
$$

That is, $r_{0}$ is the minimal number that satisfies the condition of Theorem 1.6. Let $B_{0}(G, i)$ denote the set of vertices at distance at most $r_{0}+2$ from $i$ in $G$, and note that $\left|B_{0}(G, i)\right|$ is uniformly bounded for all graphs $G \in \mathcal{B}(f, d)$.

Proof of Theorem 1.8. Denote by $U_{i}$ the event that $A_{0}^{j}=S$ for all $j \in B_{0}(G, i)$. Note that by Theorem 1.6, the event ' $Z{ }^{i}=S^{\prime}$ ' contains $U_{i}$.

Let $p_{0}<1$ be close enough to 1 so that, for any vertex $i$ in any graph $G \in \mathcal{B}(f, d)$, and any $p>p_{0}$, it holds that

$$
\mathbb{P}\left[U_{i}\right] \geq \eta>\frac{1}{2}
$$

for some $\eta$. This holds, for example, for $p_{0}>1-1 /(2 N)$, where $N$ is a uniform bound on $\left|B_{0}(G, i)\right|$.

Given a graph $G \in \mathcal{B}(f, d)$, let $I_{G} \subset V$ be a maximal set of vertices such that $B_{0}(G, i) \cap B_{0}(G, j)=\emptyset$ for all $i, j \in I_{G}$. Then the events $\left\{U_{i}\right\}_{i \in I_{G}}$ are independent, since the events ' $A_{0}^{i}=S^{\prime}$ ' are independent.

We first consider the case of finite graphs. Let $\left\{G_{n}\right\}_{n \in \mathbb{N}}$ in $\mathcal{B}(f, d)$ be a sequence of graphs such that $\lim _{n}\left|V_{n}\right|=\infty$, and let $I_{n}=I_{G_{n}}$. Note that $\lim _{n \rightarrow \infty}\left|I_{n}\right|=\infty$, since the diameters of $G_{n}$ tend to infinity.

Since $\mathbb{P}\left[U_{i}\right] \geq \eta$, and since the events $U_{i}$ are independent, then by the Chernoff bound we have that

$$
\mathbb{P}\left[\sum_{i \in I_{n}} \mathbb{1}_{U_{i}}<\left|I_{n}\right| / 2\right] \leq e^{-\frac{(\eta-1 / 2)^{2}}{2 \eta}\left|I_{n}\right|},
$$

and so, since the event ' $Z^{i}=S$ ' contains $U_{i}$, we have that

$$
\mathbb{P}\left[\hat{S}_{n} \neq S\right] \leq e^{-\frac{(\eta-1 / 2)^{2}}{2 \eta}\left|I_{n}\right|},
$$

for

$$
\hat{S}_{n}=\operatorname{sgn} \sum_{i \in I_{n}} Z^{i} .
$$

Since $\lim _{n \rightarrow \infty}\left|I_{n}\right|=\infty$ it follows that

$$
\lim _{n} \delta\left(G_{n}, p\right)=0,
$$

and we have proved the claim for finite graphs.

Let $G=(V, E) \in \mathcal{B}(f, d)$ be an infinite graph. Then, since the diameter of $G$ is infinite, there exists for every $n$ a set $I_{n} \subset V$ of size $n$ such that $B_{0}(G, i) \cap B_{0}(G, j)=$ $\emptyset$ for all $i, j \in I_{n}$. By the same reasoning as above, we have that

$$
\mathbb{P}\left[\hat{S}_{n} \neq S\right] \leq e^{-\frac{(\eta-1 / 2)^{2}}{2 \eta} n},
$$


for

$$
\hat{S}_{n}=\operatorname{sgn} \sum_{i \in I_{n}} Z^{i}
$$

and so

$$
\delta(G, p)=0 .
$$

3.7. Convergence on a non-slow growth graph. In this section we provide an example of a $d$-regular graph for which $\mathrm{M}_{d}[G, i]=\infty$ for all $i \in V$, but still the opinion of every agent converges, in the synchronous model, to period at most two, for every set of initial opinions.

Given an odd $d \geq 3$, denote by $H$ the complete bipartite graph $K_{d, d}$ on $2 d$ vertices, without a single edge $\{a, b\}$. Let $G^{\prime}$ be any $d$-regular graph. Let $F$ be any set of edges whose removal separates $G^{\prime}$ into finite components. Consider the following process. For an edge $e=\{i, j\} \in F$, remove $e$ from $G^{\prime}$, add a copy $H_{e}$ of $H$, connect $i$ to $a_{e}$ (the vertex in $H_{e}$ which corresponds to $a$ ), and connect $j$ to $b_{e}$. Repeat this process for all $e \in F$. The resulting graph will be denoted by $G$.

Lemma 3.10. For any initial choice of opinions for $G$, the opinions of all vertices converge, in the synchronous model, to period at most two.

Since $G^{\prime}$ was arbitrary, one can easily construct such graphs $G$ with $\mathrm{M}_{d}[G, i]=$ $\infty$.

Proof. We first show that for any copy of $H$ the opinions converge at time at most $t=2$ to a period at most two. Fix a copy of $H$, call it also $H$.

Indeed, if we call one side of $H A$ and the other $B$, so that $a \in A, b \in B$, then at time $t=1$ all vertices of $A \backslash\{a\}$ have value $m_{B}$, the majority of values in $B$, and similarly vertices in $B \backslash\{b\}$ have value $m_{A}$. As $d \geq 3$, both $a$ and $b$ have more neighbors in $H$ than in $G \backslash H$. Thus, at time $t=2$ all $A$ have opinions $m_{A}$, and all $B$ have $m_{B}$. Now it is easy to see that for this subgraph we have converged to a period of length at most two.

Since when we remove the copies of $H$ from $G$ we get a union of finite components, after time $t=2$, the processes in these components are independent and can be thought as applying the dynamics to finite graphs, with some fixed boundary conditions. Thus, the opinions in these components will also converge to a period of length at most two $[9]$.

\section{REFERENCES}

[1] Venkatesh Bala and Sanjeev Goyal, Learning from neighbours, The Review of Economic Studies 65 (1998), no. 3, 595-621.

[2] Eli Berger, Dynamic monopolies of constant size, Journal of Combinatorial Theory, Series B 83 (2001), no. 2, 191-200.

[3] Federico Camia, Emilio De Santis, and Charles Newman, Clusters and recurrence in the twodimensional zero-temperature stochastic Ising model, The Annals of Applied Probability 12 (2002), no. 2, 565-580.

[4] Emilio De Santis and Charles Newman, Convergence in energy-lowering (disordered) stochastic spin systems, Journal of statistical physics 110 (2003), no. 1-2, 431-442.

[5] Glenn Ellison and Drew Fudenberg, Rules of thumb for social learning, Journal of Political Economy (1993), 612-643. 
[6] Luiz Renato Fontes, Roerto Schonmann, and Vladas Sidoravicius, Stretched exponential fixation in stochastic Ising models at zero temperature, Communications in Mathematical Physics 228 (2002), no. 3, 495-518.

[7] Douglas Gale and Shachar Kariv, Bayesian learning in social networks, Games and Economic Behavior 45 (2003), no. 2, 329-346.

[8] Yuval Ginosar and Ron Holzman, The majority action on infinite graphs: strings and puppets, Discrete Mathematics 215 (2000), no. 1-3, 59-72.

[9] Eric Goles and Jorge Olivos, Periodic behaviour of generalized threshold functions, Discrete Mathematics 30 (1980), no. 2, $187-189$.

[10] C. Douglas Howard, Zero-temperature Ising spin dynamics on the homogeneous tree of degree three, Journal of Applied Probability (2000), 736-747.

[11] Yashodhan Kanoria and Andrea Montanari, Majority dynamics on trees and the dynamic cavity method, The Annals of Applied Probability 21 (2011), no. 5, 1694-1748.

[12] David Kempe, Alin Dobra, and Johannes Gehrke, Gossip-based computation of aggregate information, Proceedings of the 44th annual symposium on foundations of computer science, 2003, pp. 482-491.

[13] Warren McCulloch and Walter Pitts, A logical calculus of the ideas immanent in nervous activity, The Bulletin of Mathematical Biophysics 5 (1943), no. 4, 115-133.

[14] Gadi Moran, On the period-two-property of the majority operator in infinite graphs, Transactions of the American Mathematical Society 347 (1995), no. 5, 1649-1667.

[15] Elchanan Mossel, Joe Neeman, and Omer Tamuz, Majority dynamics and aggregation of information in social networks, Autonomous Agents and Multi-Agent Systems 28 (2014), no. 3, 408-429.

[16] Elchanan Mossel, Allan Sly, and Omer Tamuz, Strategic learning and the topology of social networks, ArXiv e-prints (August 2012), available at 1209.5527

[17] David Peleg, Size bounds for dynamic monopolies, Discrete Applied Mathematics 86 (1998), no. 2, 263-273.

[18] Devavrat Shah, Gossip algorithms, Foundations and Trends in Networking 3 (2009), no. 1, $1-125$.

[19] Ran Tessler and Oren Louidor, Geometry and Dynamics in Zero Temperature Statistical Mechanics Models, ArXiv e-prints (August 2010), available at 1008.5279.

(O. Tamuz) Weizmann Institute of Science, Faculty of Mathematics and Computer Science, Rehovot, IsRael.

E-mail address, O. Tamuz: omer.tamuz@weizmann.ac.il

(R.J. Tessler) Hebrew University, Einstein Institute of Mathematics, Jerusalem, IsRAEL.

E-mail address, R.J. Tessler: ran.tessler@mail.huji.ac.il 